\title{
Implications of Peripheral Muscular and Anatomical Development for the Acquisition of Lingual Control for Speech Production: A Review
}

\author{
Margaret Denny Richard S. McGowan \\ CReSS LLC, Lexington, Mass., USA
}

\section{Key Words}

Development $\cdot$ Tongue $\cdot$ Vocal tract $\cdot$ Child $\cdot$ Speech motor control

\begin{abstract}
Objectives: Normally developing children learn to produce intelligible speech during rapid, non-uniform growth of their articulators and other vocal tract structures. The purpose of this review is to focus attention on the consequences of peripheral growth and development for the acquisition of lingual control for speech production. This paper (1) reviews physiological underpinnings of tongue shaping and movements that are likely to be changing in young children; (2) estimates, from previously published studies, the net consequences of growth of multiple vocal tract structures on lingual control; (3) integrates our findings with the example of [I] production, and (4) highlights areas where further investigations would be most helpful. Patients and Methods: The authors searched the literature, including the PubMed database, for studies of the development of muscle proteins, muscle fibers, and motor units of the tongue, and of the growth of the tongue, jaw, adenoids, soft and hard palates, oral and pharyngeal cavities, and the vocal tract as a whole. Conclusions: Substantial anatomical and muscular data sets focused on children from 1-4 years of age, and rigorous definitions of the tongue boundaries are needed.
\end{abstract}

Copyright $\odot 2012$ S. Karger AG, Basel

\section{KARGER}

Fax +4161306 1234

E-Mail karger@karger.ch

www.karger.com
(C) 2012 S. Karger AG, Basel

$1021-7762 / 12 / 0643-0105 \$ 38.00 / 0$

Accessible online at: www.karger.com/fpl

\section{Introduction}

Coordinated movement begins before birth and all of its substrates develop throughout the lifespan. Acquisition of broader motor capabilities in young creatures occurs in the context of neural, muscular, and skeletal growth and maturation. These observations are especially relevant to the young child, who takes on the task of producing intelligible speech with an immature neuromuscular system and a growing vocal tract that is substantially different in multiple ways from that of the adult [1-5]. So complex is the sum of all growth of the articulatory apparatus from birth to adolescence that it has aptly been termed 'anatomic restructuring' (e.g., [4]).

The major goal of this review is to present certain aspects of peripheral development, growth, and anatomic restructuring particularly relevant to the acquisition of lingual control in speech production. We proceed on two levels: first, on the muscular development of the tongue itself; second, on the growth of multiple vocal tract structures with emphasis on their combined consequences for lingual control. The two levels are integrated, and illustrated, with the example of American English [I] production in an immature vocal tract. In conclusion, we propose preliminary questions and measures that will allow us to begin to address the acquisition of lingual control in a sufficient anatomic context. 
We will argue that the articulatory apparatus of young children is less well suited to produce a complete phonemic inventory than is the case with adult speakers. We focus on changes in muscular maturation and vocal tract anatomy here because we believe they will be essential to a good understanding of the acquisition of lingual control for speech. Our emphasis is intended to complement and illuminate, not to compete with, perspectives that emphasize theories of motor control, motor learning, maturation of the central nervous system and linguistically based factors in speech acquisition (e.g., [6]). Although we focus here on the development of anatomically normal children, we believe that our approach will be especially useful for understanding the speech of children with cleft palate and other craniofacial abnormalities as well.

Acoustic consequences of tongue shape and movement absolutely depend on the vocal tract in which the tongue operates; for example, they depend on the relative sizes and shapes of the tongue and mandible and the shape of the pharynx. Appropriate shaping and positioning of the entire tongue are essential to creating the patterns of constrictions, or contacts, and cavities needed to produce intelligible speech; thus, the dimensions of the whole tongue compared to those of the oral and pharyngeal cavities are of great interest. For these reasons, we believe that the development of lingual control must be explicitly considered in context of the constraints imposed by overall vocal tract development.

At present, significant gaps in our knowledge of vocal tract development remain. With the goals of framing and motivating explicit questions for further research, we review studies that relate phases of muscular maturation to movement capabilities in young animals, but comparable studies have not been done in humans. There are comparisons of the muscular development in newborn to adult humans, but very little data on young children. Many studies have measured the growth of structures or areas of the vocal tract, such as the jaw or the palate, over some period of development, and there are excellent reviews of this literature (e.g., $[3,7,8])$. To date, no one has made simultaneous measures of the multiple structures most relevant to lingual control in speech, in a reasonably sized sample of children, over the period in which speech begins to emerge until the phonemic inventory is substantially complete. For the purpose of clarity, that period is here considered to be approximately 1-4 years of age, although speech skills continue to be refined through adolescence $[9,10]$.

Evidence from studies of children's speech [11] and handwriting [12] indicate that children begin to acquire fine motor skills by adopting a 'relatively large-amplitude, low-velocity movement strategy' [11], possibly to maximize sensory information and the time available to process that information. For an unconstrained hand and arm, the same letter may be written in a wide range of sizes; for test utterances such as 'Buy Bobby', a range of jaw opening displacements is allowable. In contrast, if the tongue is to achieve highly specified trajectories or shapes within the vocal tract, boundaries of the tract such as palate and mandibular height, and pharyngeal depth, often limit children's ability to enlarge movement for ease of execution. Further motivation for this review comes from work by Gibbon, Cheng and their colleagues $[13,14]$ who have used electropalatography (EPG) to study the development of lingual control. These researchers found that undifferentiated lingual gestures, defined as unusually large areas of tongue-to-palate contact $[13,14]$, are widespread in children whose speech development is delayed or disordered [14-16]. Gibbon, Cheng, and colleagues argue that undifferentiated lingual gestures occur when speakers have not mastered the relatively independent control of the tip/blade, body, and lateral margins of the tongue seen in normal speakers of the same age. Although EPG has not been performed on children younger than 6 years of age, presumably because of technical limitations and limits of younger children's tolerance for the technology, Gibbon, Cheng, and colleagues suggest that undifferentiated lingual gestures are likely to be common in the speech of children younger than those they have tested with EPG. This review offers some insights into possible factors that may contribute to the relatively late acquisition of this independent lingual control for speech.

As noted above, the information reviewed will be integrated to examine the production of American English [I] with a young child's vocal tract. There are many vocal tract configurations that can be used to produce [I] [1720]. In particular, the tongue shapes used by various speakers are markedly diverse [19, 20]. Nevertheless, American English [I] typically requires simultaneous tongue constrictions to be produced at two levels of the tract: mid palatal and pharyngeal [17]. These requirements are particularly strong for $[\mathrm{I}]$ in initial position. The requirement for two simultaneous constrictions made with the tongue is rare in American English, and it is difficult to see how it could be satisfied without relatively independent control of the tongue's subregions as discussed above. Note that while the precise places and degrees of these constrictions may vary and trade off with each other $[19,20]$, as well as with the degree of lip round- 
Fig. 1. A summary of the requirements for an intelligible $[\mathrm{I}]$. The appropriate commands to the muscles must shape the immature vocal tract into a reasonable approximation of an acoustic tube with three constrictions and three volumes, in order to produce an acoustic output with low first, second, and third formants. This review focuses on the reasons why an immature vocal tract may not be well suited to approximate an acoustic tube with three constrictions and three volumes.

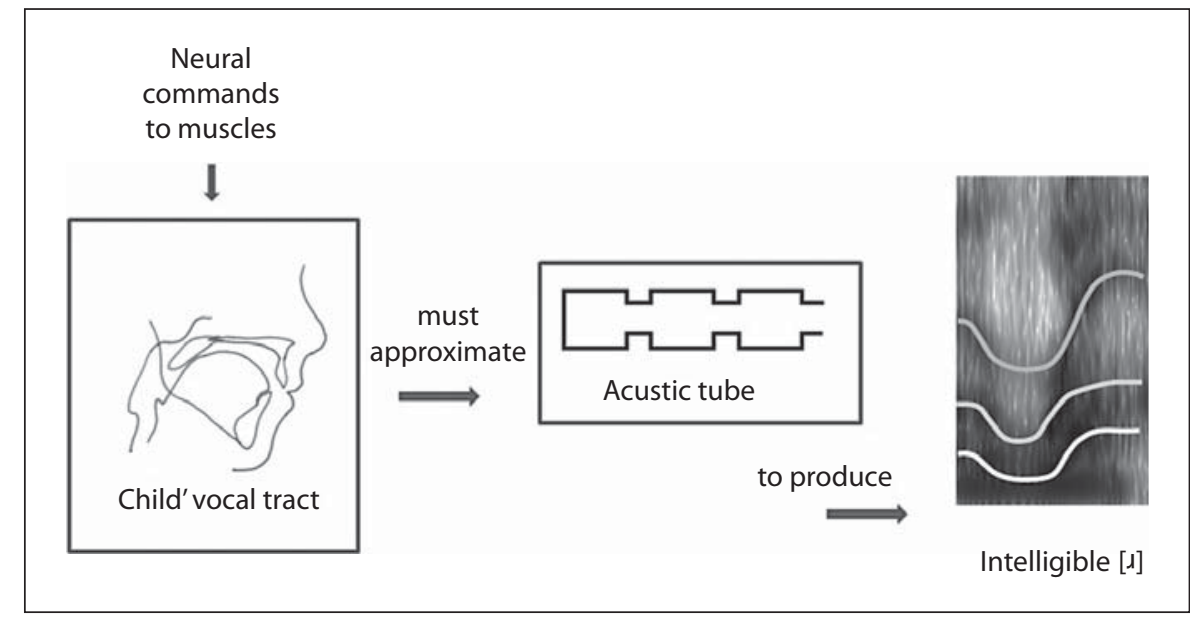

ing, the requirement for two constrictions made with the tongue remains. Our arguments about the challenges [I] poses for young children do not depend on the variable details of its articulation, or the variety of tongue shapes that can be used to achieve the required constrictions, but on the invariant requirements for an intelligible production. The protracted period of acquisition and relatively late mastery of $[\mathrm{I}]$ are also consistent with the view that there are underlying physiological and anatomical constraints on young children that make adult-like production of this phone difficult (for review, see [21]). Frequency of occurrence in a language can affect the timing of acquisition of speech sounds [22], but given the frequency of occurrence of [I] in American English [23], this is an unlikely explanation for its late mastery.

In summary, we review some physiological underpinnings of tongue shaping and movements that are likely to be changing in the young child, but have not been investigated in children. We place the child's tongue in its context by reviewing the growth of the other vocal tract structures most relevant to lingual control for speech. We integrate and illustrate this information with the example of American English [I] , and conclude by posing fundamental questions for future research and proposing a set of measures, feasible in young children with contemporary technology, that would allow us to begin to address the acquisition of lingual control in sufficient developmental and anatomic context. A graphical summary of our argument is given in figure 1 . We believe that information gained from these measures will be useful for understanding the acquisition of speech from a wide variety of theoretical perspectives.

Peripheral Muscular and Anatomical Development in Lingual Control

\section{Muscular Structure of the Tongue}

Insight into the production of complex movements can be gained by considering the capabilities of the muscles used [24-26]. In general, the human tongue is composed mostly of fast (type II) muscle fibers [27-30]. Genioglossus and the intrinsic tongue muscles are non-uniform in their spatial distributions of fiber types and sizes in a variety of non-human animals (for review, see [31]) and in humans $[27,28,32]$. The proportion of fiber types varies from anterior to posterior, such that faster-type fibers are found anteriorly and slower-type fibers posteriorly. Within fiber types, there is a gradient of muscle fiber sizes such that smaller fibers are found in the anterior tongue and larger fibers in the posterior tongue. In other words, slow muscle fibers are less common and smaller in the anterior tongue; they are more common and larger in the posterior tongue. Fast muscle fibers are more common and smaller in the anterior tongue; they are less common and larger in the posterior tongue.

The tongue muscles of the fetus and neonate are adultlike in their arrangement and general appearance $[8,33-$ 35] with two important exceptions [36]. The neonatal superior longitudinal is not well developed, particularly near the midline where it is relatively thick in the adult; this leads to a flat rather than a curved tongue dorsum as seen in the adult. Also the cross-sectional areas of the extrinsic muscles are 'considerably larger' [36] compared to the areas of the intrinsic muscles in the neonate.

The relatively undeveloped superior longitudinal in the neonate is of particular interest. In both rats [37] and humans [38], this muscle displays an 'in-series' arrangement of motor units, allowing for great flexibility and

Folia Phoniatr Logop 2012;64:105-115 
variety in choice of activation patterns. As the name suggests, such muscle is comprised of motor units with minimally overlapping territories, in this context, along the long axis of the tongue. This organization permits precisely localized contractions. In the superior longitudinal muscle, small, in-series motor units are ideal for producing precise patterns of tongue bending in the superior-toinferior dimension [39].

All of these investigations were done on excised tongues, so the angles of insertion of the intrinsic muscles could not be studied. The angles of insertion of the extrinsic muscles change as the skull grows, affecting their mechanical actions. This is discussed below in the section on development of the head and supralaryngeal vocal tract.

\section{Development of Muscle and Motor Units}

The development of muscle and motor unit types is complex, varies across species and muscles, and is open to both genetic and phenotypic (use-related) influences throughout the lifespan [40-43]. In developing experimental animals, development of adult-like myosin isoforms, the primary motor proteins, occurs at the same time as new behaviors emerge, such as walking and eating solid food. This includes transitions from one adultlike myosin isoform to another; transitions may be from fast to slow or vice versa [44-46]. Again in developing experimental animals, behavioral interventions, such as restraint or intubation, can disrupt myosin development to some extent; behavior is disrupted in parallel $[47,48]$.

In human genioglossus, $90 \%$ of myosin isoforms are adult-like by $31-42$ weeks' gestational age [49]. $72 \%$ are fast, somewhat exceeding the estimate of $60 \%$ fast myosin isoforms in the adult [28]. The authors have been unable to discover any investigations of the development of human tongue myosin isoforms in childhood other than a study by Lloyd et al. [49]. Thus, it is not known when the adult distribution of fiber types and sizes is established. It has been reported that infants who have been intubated show deficits in coordination for feeding; the longer the intubation period, the worse the deficits [50, 51]. Attention has focused on the effects of intubation on the central nervous system, but it is likely that peripheral development is affected as well. There is evidence that longterm tracheostomy, which can limit tongue mobility, adversely affects speech development in some but not all children [52-54]. This is true whether or not neurological disorders also occur; both age at tracheostomy and the duration of intubation appear to be crucial factors [54]. Most children improve with time after decannulation $[52,53]$. It is tempting to speculate that the onsets of babbling and/or articulate speech may be accompanied and supported by developmental transitions among myosin and motor unit types.

The development of one muscle relevant to lingual control has been described in human neonates, adults, and young children: the superior constrictor of the pharynx. The posterior portion of the transverse muscle of the tongue merges with fibers of the superior pharyngeal constrictor to assist in tongue retrusion in humans [55, 56]. The superior pharyngeal constrictor of the human pharynx is unlike that of other animals investigated to date. While other animals have a single layer of predominantly fast muscle, in humans, there is a fast outer layer and a slow inner layer. Each layer is independently innervated [57]. The slow inner layer of pharyngeal muscle is not present at birth; it can be detected in 2-year-olds, but is not as well formed as in adults [57]. Development of the slow layer of the pharynx may be related to the decrease in airway collapsibility that occurs in the first year of life [58]. It is not known at what time the development of the slow layer of muscle is complete.

In summary, neonatal to adult comparisons indicate that superior longitudinal and the inner layer of the pharynx are absent or underdeveloped. We argue below that tongue bending in the sagittal plane is essential for American English [I] production. This will be more difficult when the superior longitudinal muscle is less developed. Similarly, if the superior pharyngeal constrictor is not well developed, tongue retrusion for creating a pharyngeal constriction may be more difficult.

\section{Development of the Skull and Its Relation to Other Vocal Tract Structures}

It seems reasonable to assume that children learning to speak take adults as models, at least to some degree. If that is true, the process of learning would be greatly simplified if a single, simple scale factor related children's anatomy to that of adults. In fact, the scalings between child and adult anatomy are multiple and complex, and change substantially throughout time from 1-4 years of age, and well beyond. A more complete account of the scaling mismatches between adult and child can potentially shed much light on the child's approach to acquiring speech. 
An intuitively appealing approach to the complex issue of vocal tract restructuring is to separate inquiries, reports and review sections by anatomical structure, and this is a valuable approach. But if the development of lingual control is to be well understood, the summed, or net, consequences of the growths of multiple structures must be considered. At this point such net consequences may only be roughly estimated, and we have made a preliminary attempt to do so here, to illustrate the usefulness that such integrated information would have in quantified form.

Quantitative measures of the dimensions of the developing vocal tract have become available at an ever increasing rate. It is now well established that the newborn's supralaryngeal vocal tract is less than half the length of the adult male's vocal tract and that the ratio of pharynxto-mouth length grows through early adolescence $[2,4$, 5]. Over relatively long time spans during development, say 10 years, vocal tract structure dimensions can appear to grow linearly when sample rates are low and group data are analyzed. Further, the slopes of the growth lines can appear to be nearly the same for different structures (e.g., [1]). However, when structural growth is considered longitudinally with higher sampling rates and greater detail, non-linear growth curves appear to be more appropriate, even over shorter total durations [5]. In the following, we consider various aspects of growth that can affect the relative positions and sizes of the tongue and the surrounding structures, including the pharynx, the hard palate, the hyoid and so on.

The tongue itself grows in weight and volume by a factor of around 10-13 from birth to adulthood (e.g., [3]). The changes in the linear dimensions of the tongue, length, width, and thickness are nearly equal. Seibert [59], in an autopsy study, noted that from the $30-\mathrm{cm}$ long fetus to the $140-\mathrm{cm}$ tall male adult, the mean length and width of the tongue increases by a factor of 2.3 and the thickness by a factor of 2 .

The lengthening of the pharynx is a part of a more global change in the sizes and relative positions of skull bones. The larynx and hyoid bone descend in relation to the base of the skull, and the horizontal length of the hard palate and mandible grow forward from the base, as well as downward. This growth occurs as the cervical vertebrae are lengthening in such a way that the larynx descends in relation to them [60], the hyoid remains at a fairly constant position between C3 and C4 [60, 61], and the mandibular symphysis actually becomes higher when measured with respect to the cervical vertebrae [61].

The relative positions of the mandible or hyoid and the hard and soft palates provide an indication of the space

Peripheral Muscular and Anatomical Development in Lingual Control in which the anterior part of the tongue resides. According to figure 4 in King [60], the vertical distance from the posterior nasal spine to the mandibular symphysis increases from about $35 \mathrm{~mm}$ at birth to about $50 \mathrm{~mm}$ at 16 years of age for a male. This is not enough to offset a doubling of tongue thickness. On the other hand, the increase in tongue thickness can be accommodated because the vertical distance from the posterior nasal spine and the hyoid changes from about $30-55 \mathrm{~mm}$ in the same time span. Vorperian et al. [4] confirm that the hyoid descends substantially from birth to adulthood, with an initial rapid descent over the first 18 months of life.

It is not clear what may be the best measure(s) for determining the amount of oral space available to the tongue. Candidates include the distance of the mandible from the hard palate or the hyoid bone to the hard palate, or a combination of the two distances. There appears to be some opinion that the palate-to-hyoid distance is the most important factor: 'Because during normal growth the hyoid descends from the mandible, progressively more space is provided for the base of the tongue' [61].

When the changes to the relative sizes of the tongue and the space containing the tongue in the first 2 years of life are considered, it appears that the pace of vocal tract space growth exceeds that of the tongue. Thus, the oft reported observation that the tongue of the newborn fills the oral cavity may be altered after the first few months of life. Both the hard palate horizontal length and mandible length grow rapidly in the first 2 years of life. The hard palate length grows from $60 \%$ of adult length to over $80 \%$ of its adult length and the mandible length and depth from $40 \%$ of adult values to $60 \%$ of its adult values [4]. An $\mathrm{X}$-ray study of mandibular size in female children 3-16 years of age found that growth slowed after 3 until about 10 years of age [62]. On the other hand, the tongue only grows from about $50 \%$ of its adult length at birth to $60 \%$ in the first 2 years [4]. Another important factor in the change of relative tongue and vocal tract cavity size is the change of vertical distance between the larynx and the posterior nasal spine from about $35-55 \%$ of the adult distance in the first 2 years of life. This is a large component of vertical pharynx growth [4]. As the pharynx gets longer it is able to accommodate more of the total length and volume of the tongue. In sum, the size of the tongue relative to the size of the supralaryngeal vocal tract could decrease substantially during the first 2 years of life.

As the structures surrounding the tongue change their relative positions, some extrinsic tongue muscle forces change direction. For instance, the descents of the mandible and hyoid mean that the forces developed by con- 
traction of the genioglossus and hyoglossus come to possess a relatively greater vertical component with age. In addition, as the tongue descends with respect to the base of the skull the forces generated by the styloglossus possess a larger vertical component [8]. Conversely, in the young child, the styloglossus is not as well suited to create vertical force as that of the adult. How then are children and infants able to swallow, which requires raising the tongue back? To swallow, one creates an anterior tongueto-palate contact and enlarges it posteriorly to force the bolus back towards the pharynx [63]. There are no restrictions on the configuration of the tongue anterior to the seal. Thus, any muscles that help to raise the tongue back can be recruited. Adults have been observed to use a combination of styloglossus and posterior portion of the transverse muscles to raise their tongue backs for swallowing [39]; see also discussion in [64]. It is likely that young children, when swallowing, rely less on styloglossus to raise their back tongues than adults do.

Observations of X-ray pictures reveal that the inner angle between the mouth and pharynx decreases (i.e., becomes less obtuse) during development. This is also indirectly indicated by a decreasing inner angle between the soft palate and the hard palate with age [65]. Another factor in the uvular region is lymphoidal tissue. Lymphoidal tissue in the vocal tract appears to have a non-linear growth curve, resulting in particularly large adenoidal tissue areas compared to air channel areas in the nasopharynx for many children starting at the ages of 1.5-3 years, and until 8-10 years, as seen in X-ray images [66-68]. The rapid growth of the adenoids starts after infancy [69] resulting in adenoidal tissue that is convex in the anterior, or forward, direction for children as young as 3 years, but not for adults [70]. Because the soft palate approximates the adenoidal tissue during non-nasal speech, children may have a more constricted uvular air channel during vowel and sonorant consonant production than they would otherwise. Indeed, Subtelny [71] notes that excessive adenoidal tissue can move the portion of the approximation of the tongue with the soft palate forward and downward. The soft palate itself has its most rapid increase in thickness during the first year, further restricting the uvular air channel [65]. There is other lymphoidal tissue on the surface of the tongue behind the terminal sulcus of the tongue, known as the lingual tonsil, for which we do not have growth data. Enlargement of this tissue could also alter the uvular air channel, as well as the ability of the tongue to control the shape of the air channel in the uvular region.

To summarize, the supralaryngeal vocal tract grows largely as a result of the downward and forward growth of structures, including the mandible, hard palate, and hyoid bone, in relation to the base of the skull. Bone growth's precise relation to tongue growth is not known, but it appears to outpace the growth of the tongue in the first 2 years postpartum. As a result of the fact that the skull is not fully grown for young children, the extrinsic tongue muscles are less effective at moving the tongue in a vertical direction. The growth of the adenoids can have a significant effect on the oral air channel in the uvular region from ages 3-8 years. Much of what can be gleaned from the literature on vocal tract growth is either of a qualitative nature or of an approximate quantitative nature, although recent researchers are making progress in providing more precise quantitative measures (e.g., [5]).

\section{Putting It All Together: Producing American English}

[I]

We integrate and summarize our findings by examining their consequences for children's attempts to produce the American English prevocalic [I]. The phone [I] is generally articulated without a marked steady-state interval [72]. However, the tongue shapes and positions appropriate for the production of the acoustic characteristics of [I], such as low third formant frequency and close second and third formant frequencies, are attained for some time interval. The shapes and positions of the tongue that produce such acoustic characteristics during $[\mathrm{I}]$ production are called [I]-like articulations here.

As discussed in the introduction, an [I]-like articulation requires simultaneous tongue constrictions that are produced at two levels of the tract: mid-palatal and pharyngeal with a distinct cavity between constrictions. For any given production of $[\mathrm{I}]$, the tongue configuration with the narrowest constrictions is called the extreme [I]like configuration. To make the example more concrete, syllable initial $[\mathrm{I}]$ starts at its most extreme [I]-like articulation with two tongue constrictions, but the tongue immediately moves toward a vowel articulation as in a glide-vowel sequence. Figure 2 illustrates the points of discussion for the particular case of retroflex [I], though the arguments below can also be applied to tonguebunched [I].

In the present work we are concerned with the snapshot of the extreme $[\mathrm{I}]$-like articulation in the trajectory of the tongue shaping. While the tongue is moving and changing shape, there is an interval of time where the tongue is close to the extreme $[\mathrm{I}]$-like articulation. Thus, it is important to consider the snapshots of extreme $[\mathrm{I}]$ - 
Fig. 2. Cartoon of child's tongue (right) as it might be shaped for [I]. The angle $\theta$ between the palate and the pharynx is more obtuse, the adenoids are enlarged, and there is more of a dimple in the tongue between the two oral constrictions for the child than for the adult (left).

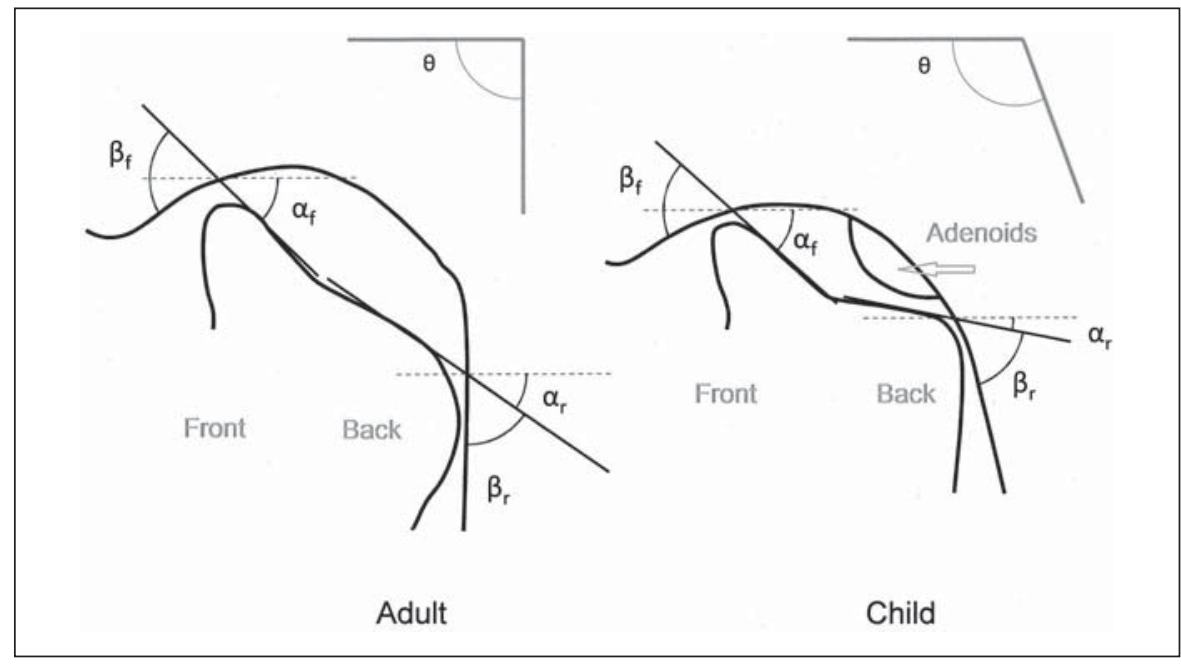

like articulations as part of the motor control necessary for the articulation of the $[\mathrm{I}]$ segment.

The anatomical differences between children and adults have consequences for their articulation of [x]. Because the pharynx is proportionately shorter in children than adults, the rear constriction needs to be closer to the uvula in children than in adults in order to maintain the same ratio of acoustic tube lengths. The adenoids could be obstructing the uvular channel for children. Control of the tongue shape is further complicated by the different orientations of some extrinsic muscles in children as discussed above. In a model proposed by Maeda and Honda [73], which is based on EMG measures [74], the major agonist-antagonist pairs for tongue movement are posterior genioglossus-hyoglossus and anterior genioglossus-styloglossus. The former pair moves the tongue along a front-high, low-back diagonal, and the latter pair moves the tongue along a front-low, back-high diagonal (see schematic in fig. 3). These diagonals are pictured as being approximately orthogonal for the adult. We have redrawn the adult schematic with a young child's proportion as nearly as we can estimate from available data. For the young child the diagonals would have a more frontback orientation, and thus, would not be nearly as orthogonal and would not be as effective in moving the tongue vertically. Finally, the inner angle between the rear pharyngeal wall and the hard palate (angles $\theta$ in fig. 2; again the child's schematic is an estimate) is important when two constrictions are formed: one in the oral cavity and another in the uvular region or pharyngeal cavity. This angle is supposed to be larger for the young child than the adults.

Peripheral Muscular and Anatomical Development in Lingual Control
Given the differences between young children and adults on the constraints for the formation of the tongue constrictions, it is possible to explore some of the consequences for the differences in tongue shape. Shown in figure 2 are lines tangent to the tongue's midsagittal outline behind the forward constriction and in front of the rear constriction for both the child's and the adult's vocal tract. These angles possess orientations with respect to the horizontal, and these orientations are quantified by the angles $\alpha_{\mathrm{f}}$ for the forward tangent and $\alpha_{\mathrm{r}}$ for the rear tangent. If the forward tangent intersects the palate and the rear tangent intersects the uvula/rear pharyngeal wall with the same angles, $\beta_{\mathrm{f}}$ and $\beta_{\mathrm{r}}$ for both the child and the adult, the difference between the orientation angles $\alpha_{\mathrm{f}}$ and $\alpha_{\mathrm{r}}$ must be greater for the child than for the adult. The difference in the $\alpha$ angles will increase with $\theta$. Also, the farther forward the rear constriction is, the greater the difference in the orientation angles $\alpha_{\mathrm{f}}$ and $\alpha_{\mathrm{r}}$.

Further, if an air cavity is to be formed between the two constrictions, then a 'dimple' in the midsagittal outline of the tongue may be required for children between the two constrictions because of the large $\theta$ and because of adenoidal growth. In fact, adults sometimes form such a dimple (e.g., [18]). The dimple means that the curvature of the midsagittal outline of the tongue is such that 'bowls' opening away from the palate or rear wall of the pharynx are formed at the constrictions and a bowl opening toward the uvula is formed in between the constrictions. Again, this dimple would be more pronounced the more forward the rear constriction. The radii of curvature of these bowls decrease as the dimple becomes more pronounced. Further, all the radii of curvature are smaller in

Folia Phoniatr Logop 2012;64:105-115 


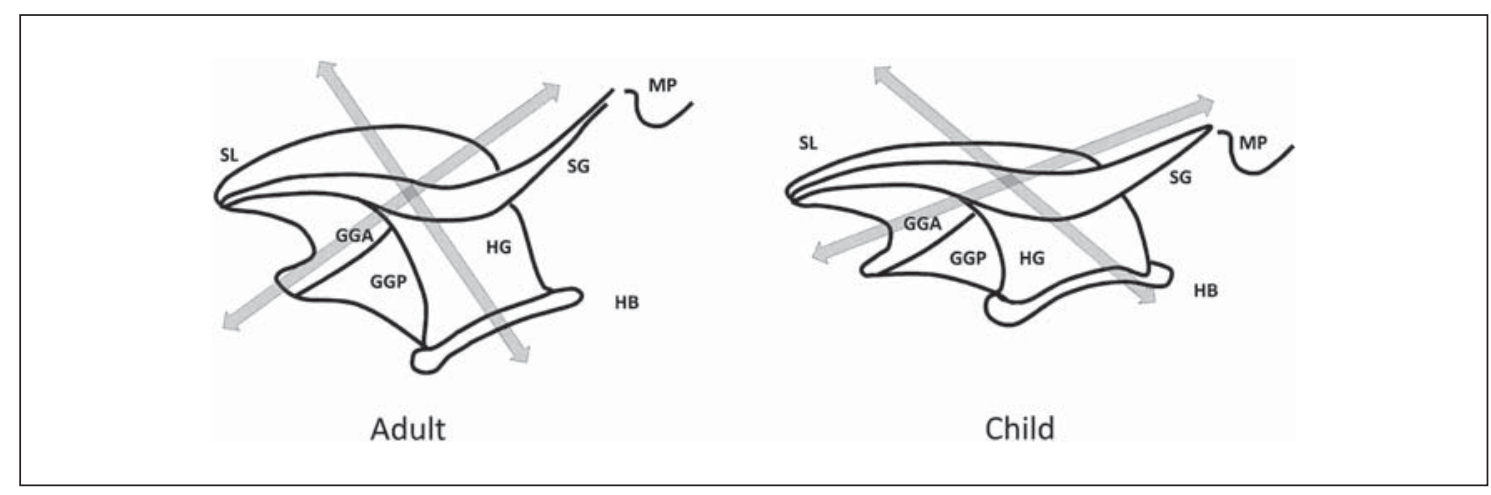

Fig. 3. Left: a cartoon (redrawn from Gray's Anatomy) showing the spatial relationships among the tongue, the mastoid process, and the hyoid bone in an adult. GGA = Anterior genioglossus; GGP = posterior genioglossus; $\mathrm{HB}=$ hyoid bone; $\mathrm{HG}=$ hyoglossus; $\mathrm{SG}=$ styloglossus; $\mathrm{SL}=$ superior longitudinal; $\mathrm{MP}=$ mastoid process. Right: a redrawing of the same structures as seen in a young child. Note the higher hyoid bone and the lower mastoid process relative to the tongue body and the less developed superior longitudinal muscle. The arrows indicate the directions of forces generated when GGP and HG are cocontracted and when GGA and SG are co-contracted. Note the less vertical arrows seen for the child (right). children than would be required of a larger, adult-sized vocal tract, if proportionate acoustic tube lengths and areas are to be maintained. Both the change of tangent orientation and radii of curvature indicate that young children need to bend the body tongue more than adults do in order to form the two constrictions. Also, while most adults may be able to produce $[\mathrm{I}]$ with two regions of small radii of curvature at the constrictions, children could require three regions of even smaller radii of curvature with the dimple. Note that throughout this discussion only ordinal comparisons have been made; the data required in order to specify the relevant adult-child differences precisely have yet to be collected.

The dimple between the anterior and posterior constrictions is most likely to require, in part, precisely localized portions of the superior longitudinal and genioglossus muscles, but the superior longitudinal is not well developed in young children. The fiber type composition of the tongue body, including the genioglossus, may be changing during the period of $[\mathrm{I}]$ acquisition. Alternatively, the relatively larger muscle fibers of the mid to posterior tongue may be established but be ill suited to creating the highly localized curvature and changes in curvature that are required. The result is that children must master a more challenging articulatory task, because of their absolute size and proportions, with a musculature that may be less well suited for that task.

The geometrical argument just presented suggests that young children would need to control their tongues more precisely than adults. Further, the review indicates that children possess a less well-developed muscular structure to use for control in $[\mathrm{I}]$ production.

As noted above, young children acquiring fine motor skills such as speech and handwriting typically produce movements that are larger than an adult's in proportion to their body size, possibly to maximize the use of sensory information [11]. The example just given makes it particularly clear, in detail, why such a strategy cannot be employed by young children to produce $[\mathrm{I}]$.

\section{Conclusion}

This review has given an overview of two areas relevant to the acquisition of lingual control for speech. First, the development of human tongue muscles in early life, and second, the net implications of changes in multiple vocal tract structures for lingual control in speech. We chose these areas for review because little is known about either of them; because it would be immensely valuable to fill these gaps in knowledge, and because it should be possible to make substantial progress here. The two areas are closely related, and we have given an example of how they can be applied together. American English [I] is a particularly clear example in applying knowledge of the muscular and anatomical constraints on production by young speakers, but the same approach may be generalized to other phonemes requiring lingual control as well. We believe that such progress can be integrated into a broad range of viewpoints on the acquisition of speech. 
We have not attempted to include all possible relevant factors, but only wish to indicate that these peripheral factors are an important component in understanding the development of speech production.

Of great concern for this work is the development of the superior longitudinal muscle; at present we know it is very little developed in the neonate but the time course of its attaining adult-like proportions to the rest of the tongue is unknown. Other major issues include the peripheral effects of intubation on the tongue, and development of muscle and motor unit types in both extrinsic and intrinsic tongue muscles. All of these inquiries are possible, though labor intensive, based on cadaver material.

Excellent overviews of multiple aspects of vocal tract growth across the lifespan are available, both as reviews (e.g., $[3,6])$ and as new investigations $[4,5]$, and we have relied on these here. Given the power of current imaging techniques (for review, see [75]), it should be possible to make real progress on the simultaneous development of the multiple vocal tract structures relevant to lingual control from ages 1-4. Children are scanned or X-rayed for a variety of reasons, many of which do not affect vocal tract growth or morphology. Some substantial data sets are already available, such as X-rays at dental schools.

Most importantly, data sets are needed that are focused on the years from birth to 4 years of age, the times when vocal tract growth is greatest and the children are mastering their phonetic inventories. Of course, the full lifespan perspective is of great interest (e.g., $[2,3,5])$ and speech motor control continues to be refined into adolescence $[9,10]$. We simply point out that the rapidity and complexity of vocal tract growth during early speech acquisition will require the collection of substantial data sets specific to this age group.

Specifying the dimensions of the tongue alone will be a substantial research project, but one that will be necessary for a good understanding of its control in speech. There are both theoretical and practical issues to be addressed. Theoretical issues involve the boundaries of the tongue to be measured. It will be necessary to develop precise definitions of length, width, and depth of the tongue. The tongue is not a rectangular slab (e.g., [64]), so more detailed dimensions of the tongue based on actual lingual geometry need to be developed. In addition, it is not clear how the extrinsic tongue muscles should be treated when measuring the dimensions of the tongue. This is particularly important where the genioglossus muscle is concerned. Practical issues center on collection of normative data sets. MRI scans would be ideal and there is precedent for the organization of large-scale data collection in young children [76].
An intuitively appealing approach is to organize inquiries around particular gestures or phonemes of interest. Here, we continue our example by considering those measures that would be most helpful to testing our reasoning about the challenges posed by $[\mathrm{I}]$ production in young children. We further limit our discussion to measures that could be made from dental X-rays already available.

It was indicated above that the angle between the mouth and the pharynx would be a measure of great value. However, with continuously changing direction in the vocal tract axis, this angle may be ill defined. A well-defined parametric function that could replace this is the rate of change in the direction of the vocal tract axis in the oropharyngeal region. That rate of change would be with respect to normalized vocal tract length. The location and dimensions of the adenoids and tonsils can be characterized in detail as well. Though frequently overlooked, these lymphoid tissues likely affect the ability of the tongue to form constrictions in the oral cavity, as discussed above.

The growth of the skull relevant to lingual control can also be measured from X-ray images. Some common landmarks are needed as reference points for the complex three-dimensional growth patterns of the skull and vocal tract in young children. In a lateral view, we suggest the posterior clinoid process, the anterior base of the styloid process, the most anterior and posterior points of the body of the mandible, the most superior margin of the condyle of the mandible, and the posterior nasal spine of the hard palate, and the anterosuperior border of the hyoid bone. The posterior clinoid process on the base of the skull provides a common reference point for the growth of the other structures.

For some aspects of speech acquisition, the unsuitability of many techniques for use in babies and young children continues to impose rigorous limits on direct investigations. Modeling is an appealing alternative, and promising work has been done in this area. Unfortunately, given the lack of data on children, these models have had to rely on adult specifications [77], which limits their usefulness. Not until we possess rich data sets based on development of the relevant muscular and other vocal tract physiology and anatomy, will it be possible to rigorously model speech development in children too young to scan [78].

\section{Acknowledgements}

This work was supported by grant NIDCD-001247 to CReSS LLC. The authors thank Rebecca W. McGowan and anonymous reviewers for reviewing and helping to improve the manuscript. 


\section{References}

$\checkmark 1$ Arens R, McDonough JM, Corbin AM, Hernandez ME, Maislin G, Schwab RJ, Pack AI: Linear dimensions of the upper airway structure during development: assessment by magnetic resonance imaging. Am J Respir Crit Care Med 2002;165:117-122.

$>2$ Fitch WT, Giedd J: Morphology and development of the human vocal tract: a study using magnetic resonance imaging. J Acoust Soc Am 1999;106:1511-1521.

$>3$ Kent RD, Vorperian HK: Development of the craniofacial-oral-laryngeal anatomy: a review. J Med Speech Lang Pathol 1995;3:145190.

-4 Vorperian HK, Kent RD, Lindstrom MJ, Kalina CM, Gentry LR, Yandell BS: Development of vocal tract length during early childhood: a magnetic resonance imaging study. J Acoust Soc Am 2005; 117:338-350.

$>5$ Vorperian HK, Wang S, Chung MK, Schimek EM, Durtschi RB, Kent RD, Ziegert AJ, Gentry LR: Anatomic development of the oral and pharyngeal portions of the vocal tract: an imaging study. J Acoust Soc Am 2009;125: 1666-1678.

6 Hardcastle WJ, Laver J, Gibbon FE (eds): The Handbook of Phonetic Sciences, ed 2. Oxford, Wiley-Blackwell, 2010.

7 Beck JM: Organic variation of the vocal apparatus; in Hardcastle WJ, Laver J, Gibbon FE (eds): The Handbook of Phonetic Sciences. Oxford, Wiley-Blackwell, 2010, pp 155-201.

8 Bosma JF: Anatomy of the Infant Head (Johns Hopkins Series in Contemporary Medicine and Public Health). Baltimore, Johns Hopkins University Press, 1986.

$\checkmark 9$ Sadagopan N, Smith A: Developmental changes in the effects of utterance length and complexity on speech movement variability. J Speech Lang Hear Res 2008;51:1138-1151.

$\checkmark 10$ Walsh B, Smith A: Articulatory movements in adolescents: evidence for protracted development of speech motor control processes. J Speech Lang Hear Res 2002;45:11191133.

-11 Riely RR, Smith A: Speech movements do not scale by orofacial structure size. J Appl Physiol 2003;94:2119-2126.

$>12$ Hamstra-Bletze L, Blote AW: Development of handwriting in primary school: a longitudinal study. Percept Mot Skills 1990;70:759770 .

$\checkmark 13$ Cheng HY, Murdoch BE, Goozée JV, Scott D: Electropalatographic assessment of tongueto-palate contact patterns and variability in children, adolescents, and adults. J Speech Lang Hear Res 2007;50:375-392.

14 Gibbon FE: Undifferentiated lingual gestures in children with articulation/phonological disorders. J Speech Lang Hear Res 1999;42:382-397.

15 Gibbon FE, Wood SE: Articulatory drift in the speech of children with articulation and phonological disorders. Percept Mot Skills 2002;95:295-307.
16 Goozée J, Murdoch B, Ozanne A, Cheng Y, Hill A, Gibbon FE: Lingual kinematics and coordination in speech-disordered children exhibiting differentiated versus undifferentiated lingual gestures. Int J Lang Commun Disord 2007;42:703-724.

17 DeLattre P, Freeman DC: A dialect study of American r's by X-ray motion picture. Linguistics 1968;44:29-68.

-18 Zhou X, Espy-Wilson CY, Boyce S, Tiede M, Holland C, Choe A: A magnetic resonance imaging-based articulatory and acoustic study of 'retroflex' and 'bunched' American English /r/. J Acoust Soc Am 2008;123:44664481.

19 Nieto-Castanon A, Guenther FH, Perkell JS, Curtin HD: A modeling investigation of articulatory variability and acoustic stability during American English /r/ production. J Acoust Soc Am 2005; 117:3196-3212.

20 Westbury JR, Hashi M, Lindstrom MJ: Differences among speakers in lingual articulation for American English [I]. Speech Comm 1998;26:203-226.

21 McGowan RS, Nittrouer S, Manning CJ: Development of $[\mathrm{I}]$ in young, Midwestern, American children. J Acoust Soc Am 2004; 115:871-884.

22 Pye C, Ingram D, List H: A comparison of initial consonant acquisition in English and Quiche; in Nelson KE, Van Kleek A (eds): Children's Language, vol 6. Hillsdale, NJ, Erlbaum, 1987, pp 175-190.

23 Mines MA, Hanson BF, Shoup JE: Frequency of occurrence of phonemes in conversational English. Lang Speech 1978;21:221-224.

24 Lieber RL, Frieden J: Functional and clinical significance of skeletal muscle architecture. Muscle Nerve 2000;23:1647-1666.

25 Monti RJ, Roy RR, Edgerton VR: Role of motor unit structure in defining function. Muscle Nerve 2001;24:848-866.

26 Sciote JJ, Morris TJ: Skeletal muscle function and fibre types: the relationship between occlusal function and the phenotype of jawclosing muscles in human. J Orthod 2000;27: 15-30.

-27 Stål P, Marklund S, Thornell LE, De Paul R, Eriksson PO: Fibre composition of human intrinsic tongue muscles. Cells Tissues Organs 2003;173:147-161.

-28 Saigusa H, Niimi S, Yamashita K, Gotoh T, Kumada M: Morphological and histochemical studies of the genioglossus muscle. Ann Otol Rhinol Laryngol 2001;110: 779-784.

29 Sokoloff, AJ, Daugherty M, Li H: Myosin heavy-chain composition of the human hyoglossus muscle. Dysphagia 2010;25:81-93.

30 Sokoloff AJ, Yang B, Li H, Burkholder TJ: Immunohistochemical characterization of slow and fast myosin heavy chain compositions of muscle fibers in the styloglossus muscle of the human and macaque. Arch Oral Biol 2007;52:533-543.
31 Smith JC, Goldberg SJ, Shall MS: Phenotype and contractile properties of mammalian tongue muscles innervated by the hypoglossal nerve. Respir Physiol Neurobiol 2005; 147: 253-262.

-32 Granberg I, Lindell B, Eriksson PO, PedrosaDomellöf F, Stål P: Capillary supply in relation to myosin heavy chain fibre composition of human intrinsic tongue muscles. Cells Tissues Organs 2010;192:303-313.

33 Bell WA: Muscle patterns of the late fetal tongue tip. Angle Orthod 1970;40:262-265.

34 Cooper S: Muscle spindles in the intrinsic muscles of the human tongue. J Physiol 1953; 122:193-202.

35 Touré G, Vacher C: Anatomic study of tongue architecture based on fetal histological sections. Surg Radiol Anat 2006;28:547552 .

-36 Iskander A, Sanders I: Morphological comparison between neonatal and adult human tongues. Ann Otol Rhinol Laryngol 2003; 112:768-776.

37 Sokoloff AJ: Localization and contractile properties of intrinsic longitudinal motor units of the rat tongue. J Neurophysiol 2000; 84:827-835.

38 Slaughter K, Li H, Sokoloff AJ: Neuromuscular organization of the superior longitudinalis muscle in the human tongue. Cells Tissues Organs 2005;181:51-64.

39 Napadow VJ, Chen Q, Wedeen Van J, Gilbert RJ: Biomechanical basis for lingual muscular deformation during swallowing. Am J Physiol 1999;277(3 Pt 1):G695-G701.

40 Brozanski BS, Guthrie RD, Volk EA, Cameron WE: Postnatal growth of genioglossal motoneurons. Pediatr Pulmonol 1989;7: 133-139.

41 Cameron WE, Fang H, Brozanski BS, Guthrie RD: The postnatal growth of motoneurons at three levels of the cat neuraxis. Neurosci Lett 1989;104:274-280.

42 Navarrette R, Vrbova G: Activity-dependent interactions between motoneurones and muscles: their role in the development of the motor unit. Prog Neurobiol 1993;41:93-124.

43 Núñez-Abades PA, Cameron WE: Morphology of developing rat genioglossal motoneurons studied in vitro: relative changes in diameter and surface area of somata and dendrites. J Comp Neurol 1995;353:129-142.

44 Korfage JAM, van Wessel T, Langenbach GEJ, Ay F, van Eijden TMGJ: Postnatal transitions in myosin heavy chain isoforms of the rabbit superficial masseter and digastric muscle. J Anat 2006;208:743-751.

45 Lowrie MB, More AF, Vrbová G: The effect of load on the phenotype of the developing rat soleus muscle. Pflugers Arch 1989;415: 204-208.

46 Navarrette R, Vrbova G: Changes of activity patterns in slow and fast muscles during postnatal development. Dev Brain Res 1983; 8:11-19. 
-47 Kinirons SA, Shall MS, McClung JR, Goldberg SJ: Effect of artificial rearing on the contractile properties and myosin heavy chain isoforms of developing rat tongue musculature. J Neurophysiol 2003;90:120-127.

48 Smith JC, Moore WA, Goldberg SJ, Shall MS: Contractile properties and myosin heavy chain composition of rat tongue retrusor musculature show changes in early adulthood after 19 days of artificial rearing. J Appl Physiol 2006; 101:1053-1059.

49 Lloyd JS, Brozanski BS, Daood M, Watchko JF: Developmental transitions in the myosin heavy chain phenotype of human respiratory muscle. Biol Neonate 1996;69:67-75.

-50 Bier JA, Ferguson A, Cho C, Oh W, Vohr BR: The oral motor development of low-birthweight infants who underwent orotracheal intubation during the neonatal period. Am J Dis Child 1993;147:858-862.

-51 Poore M, Barlow SM, Wang J, Estep M, Lee J: Respiratory treatment history predicts suck pattern stability in preterm infants. J Neonatal Nurs 2008;14:185-192.

52 Kamen RS, Watson BC: Effects of long-term tracheostomy on spectral characteristics of vowel production. J Speech Hear Res 1991; 34:1057-1065.

53 Kertoy MK, Guest CM, Quart CM, Lieh-Lai M: Speech and phonological characteristics of individual children with a history of tracheostomy. J Speech Lang Hear Res 1999;42: 621-635.

54 Jiang D, Morrison GA: The influence of long-term tracheostomy on speech and language development in children. Int J Pediatr Otorhinolaryngol 2003;67(suppl 1):S217S220.

- 55 Kokawa T, Saigusa H, Aino I, Matsuoka C, Nakamura T, Tanuma K, Yamashita K, Niimi S: Physiological studies of retrusive movement of the human tongue. J Voice 2006;3:414-422.
Saigusa H, Yamashita K, Tanuma K, Saigusa M, Niimi S: Morphological studies for retrusive movement of the human adult tongue. Clin Anat 2004;17:93-98.

$57 \mathrm{Mu}$ M, Sanders I: Neuromuscular specializations within human pharyngeal constrictor muscles. Ann Otol Rhinol Laryngol 2007; 116:604-617.

58 Isono S, TanakaA, Ishikawa T, Nishino T: Developmental changes in collapsibility of the passive pharynx during infancy. Am J Respir Crit Care Med 2000;162:832-836.

59 Seibert JR: A morphometric study of normal and abnormal fetal to childhood tongue size. Arch Oral Biol 1985;30:433-430.

60 King EW: A roentgenographic study of pharyngeal growth. Angle Orthod 1952;22:23-37.

61 Tourné LPM: Growth of the pharynx and its physiologic implications. Am J Orthod Dentofacial Orthop 1991;132:129-139.

62 Tracy WE, Savara BS: Norms of size and annual increments of five anatomical measures of the mandible in girls 3 to 16 years of age. Arch Oral Biol 1966;11:587-598.

63 Hiiemae KM, Palmer JB: Tongue movements in feeding and speech. Crit Rev Oral Biol Med 2003;14:413-429.

64 Stone M, Faber A, Raphael LJ, Shawker TH: Cross-sectional tongue shape and linguapalatal contact patterns in [s], [I] , and [1]. J Phon 1992;20:253-270.

65 Subtelny JD: A cephalometric study of the growth of the soft palate. Plast Reconstr Surg 1957;19:49-62.

66 Fujioka M, Young LW, Girdany BR: Radiographic evaluation of adenoidal size in children: adenoidal-nasopharyngeal ratio. AJR Am J Roentgenol 1979;133:401-404.

67 Handelman CS, Osborne G: Growth of the nasopharynx and adenoid development from one to eighteen years. Angle Orthod 1976;46:253-259.

68 Pruzansky S: Roentgencephalometric studies of tonsils and adenoids in normal and pathologic states. Ann Otol Rhinol Laryngol 1975;84:55-62.
69 Capitanio MA, Kirkpatrick JA: Nasopharyngeal lymphoidal tissue: Roentgen observations in 257 children two years of age or less. Radiology 1970;96:389-391.

70 Jeans WD, Fernando DC, Maw AR, Leighton BC: A longitudinal study of growth of the nasopharynx and its contents in normal children. Br J Radiol 1981;54:117-121.

71 Subtelny JD: The significance of adenoid tissue in orthodontia. Angle Orthod 1954;24: 59-69.

72 Stevens KN: Acoustic Phonetics. The MIT Press, Cambridge, MI, 1998.

73 Maeda S, Honda K: From EMG to formant patterns of vowels: the implications of vowel spaces. Phonetica 1994;51:17-29.

74 Baer T, Alfonso PJ, Honda K: Electromyography of the tongue muscles during vowels in /xpıp/ environment. Annual Bulletin of the Research Institute of Logopedics and Phoniatrics 1988;22:7-19.

75 Stone M: Laboratory techniques for investigating speech articulation; in Hardcastle WJ Laver J, Gibbon FE (eds): The Handbook of Phonetic Sciences. Oxford, Wiley-Blackwell, 2010, pp 155-201.

76 Almli CR, Rivkin MJ, McKinistry RC: The NIH MRI study of normal brain development (Objective-2): newborns, infants, toddlers, and preschoolers. NeuroImage 2007; 35:308-325.

77 Menard L, Schwartz JL, Boë LJ, Aubin J: Articulatory-acoustic relationships during vocal tract growth for French vowels: analysis of real data and simulations with an articulatory model. J Phon 2007;35:1-19.

-78 Mijailovich SM, Stojanovic MK, Liang A, Wedeen VJ, Gilbert, RJ: Derivation of a finite-element model of lingual deformation during swallowing from the mechanics of the mesoscale myofiber tracts obtained by MRI. J Appl Physiol 2010;109:1500-1514. 